\title{
Origin of electric field enhancement in field emission from amorphous carbon thin films
}

\author{
J. D. Carey, ${ }^{\text {a) }}$ R. D. Forrest, and S. R. P. Silva \\ School of Electronics, Computing and Mathematics, University of Surrey, Guildford GU2 7XH, \\ United Kingdom
}

(Received 12 December 2000; accepted for publication 20 February 2001)

\begin{abstract}
The observation of electron emission from amorphous carbon thin films at low applied electric fields is explained in terms of an enhancement of the field brought about by dielectric inhomogeneities within the film. These inhomogeneities originate from the differences between conductive, spatially localized $s p^{2} \mathrm{C}$ clusters surrounded by a more insulating $s p^{3}$ matrix. By a more complete understanding of the concentration and distribution of the clusters, a generic model for field emission from amorphous carbon thin films can be developed. Extensions of this model to explain the emission properties of carbon nanotubes and carbon nanocomposite materials are also presented.

(C) 2001 American Institute of Physics. [DOI: 10.1063/1.1366369]
\end{abstract}

The possible use of amorphous carbon $(a-\mathrm{C})$ and hydrogenated amorphous carbon $(a-\mathrm{C}: \mathrm{H})$ based materials as cold cathodes has now been well documented. ${ }^{1,2}$ To date there have been numerous reports of field emission (FE) at low macroscopic electric fields from a range of $a-\mathrm{C}$ and $a-\mathrm{C}: \mathrm{H}$ based materials. ${ }^{1-4}$ In the case of emission from diamondlike carbon (DLC) films it has been reported that, the main barrier which controls emission may lie at the front film/ vacuum interface. ${ }^{3}$ By contrast, for low defect density polymeric-like carbon (PAC) $a-\mathrm{C}: \mathrm{H}$ films the presence of a heterojunction at the $a-\mathrm{C}: \mathrm{H} / \mathrm{Si}$ rear contact, resulting in hot electron transport through the film has been proposed. ${ }^{4}$ This model was used to explain the observed strong dependence of the threshold field $\left(E_{\mathrm{th}}\right)$ with film thickness. ${ }^{5}$ Although the different types of film possess different physical properties and emission has been explained by different mechanisms, it has been observed that emission is often highly nonuniform across the film surface. ${ }^{3,4,6}$ One of the major problems hindering the development of thin film carbon cathodes is the lack of understanding of a definitive mechanism for emission at low applied fields, typically $<20 \mathrm{~V} / \mu \mathrm{m} .{ }^{3-7}$

Emission from flat metal surfaces often requires fields in excess of $500 \mathrm{~V} / \mu \mathrm{m},{ }^{2}$ whereas previous atomic force microscopy (AFM) studies of $a-\mathrm{C}$ and $a-\mathrm{C}: \mathrm{H}$ films have indicated that the films possess rms roughnesses of less than $1 \mathrm{~nm},{ }^{1-4}$ along with no evidence of surface protrusions which can act as a source of geometric field enhancement. Two recent studies by Ilie et al. ${ }^{8}$ and Carey et al. ${ }^{9}$ have proposed that it is the $s p^{2} \mathrm{C}$ cluster size and concentration that play an important role in the FE process. In the study by Ilie, an optimum cluster size for emission of 1.5-2 nm was proposed. Carey showed that for $a-\mathrm{C}: \mathrm{H}$ films deposited at different selfbiases, the FE could be explained in terms of the connectivity between $s p^{2}$ clusters. In this letter, we examine whether there is any dependence of $E_{\mathrm{th}}$ with thickness for DLC films and compare these results to the behavior reported ${ }^{5}$ for PAC films. Scanning tunneling microscope (STM) images of the DLC films, show that while the films may be atomically

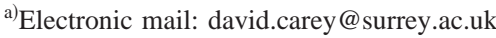

smooth they do exhibit variations in their conductivity, which are attributed to dielectric inhomogeneities within the film. Due to the shape of the field lines emanating from these inhomogeneities, we propose that they give rise to an enhancement of the electric field which plays an important role in the FE properties of these films.

$a$-C:H films were deposited on the driven electrode of a Plasma Technology DP800 capacitively coupled radio frequency $(13.56 \mathrm{MHz})$ plasma enhanced chemical vapor deposition system using $n$-type $(1-2 \Omega \mathrm{cm})(100) \mathrm{Si}$ as a substrate material. During deposition feed gases of methane $(30 \mathrm{sccm})$ with $\mathrm{He}(75 \mathrm{sccm})$ were used. The total deposition pressure was 200 mTorr and the self-bias on the driven electrode was altered by changing the rf power. Film thicknesses were measured using ellipsometry. The FE characteristics of these films were examined using a sphere-to-plane geometry in which a $5 \mathrm{~mm}$ stainless-steel ball bearing is suspended 40 $\mu \mathrm{m}$ above the surface at a high positive potential in a vacuum better than $4 \times 10^{-6}$ mbar. The high voltage is stepped up and down four times. Although a spherical anode is used the electric field between the anode and the surface of the film can be modeled as a parallel plate. Using a sphereto-plane electrode arrangement, local areas under the anode are tested rather than sampling all sites on the cathode surface as in a planar diode arrangement. Each film is tested in a number of different positions to act as a measure of the reproducibility of the emission. In some of the samples a high initial electric field is sometimes required before the onset of stable emission. This is evidence of "conditioning" of the film and in some cases is accompanied by surface changes. The threshold field is defined as the macroscopic electric field which gives an emission current of $1 \mathrm{nA}$. STM measurements were made in ambient conditions using a Digital Instrument Nanoscope IIIa employing a high resolution A scanner and using a sharpened Pt-Ir tip.

In order to investigate the possible FE mechanisms present in these films, a series of films of different thickness were grown at self-biases of -90 and $-265 \mathrm{~V}$. This complements the work presented in an earlier paper for a series of low bias (approximately $-10 \mathrm{~V}$ ) PAC films. ${ }^{5}$ The variation 


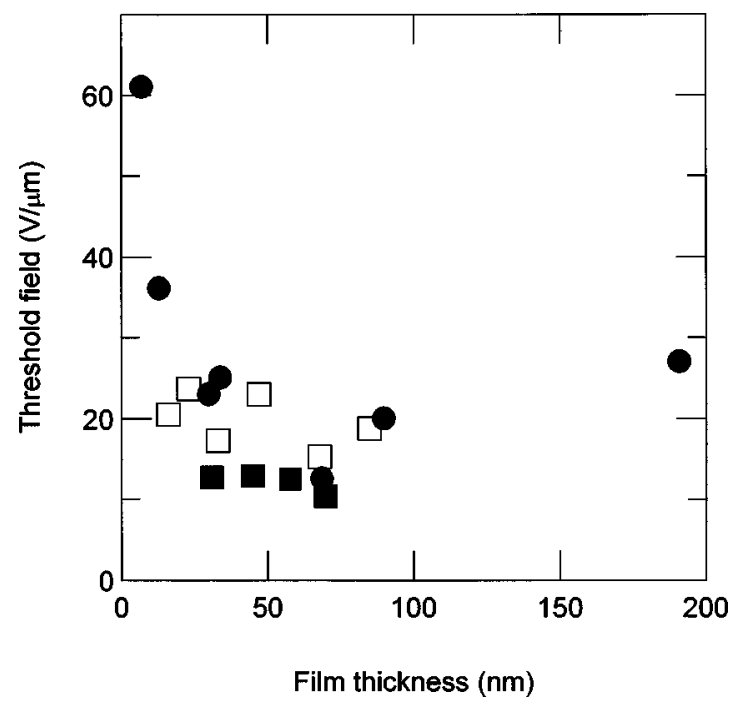

FIG. 1. Variation of threshold field against film thickness for DLC films deposited at $-90 \mathrm{~V}(\square)$ and $-265 \mathrm{~V}(\mathbf{\square})$ self-bias. The data for the PAC films (๑) reported in Ref. 5 are also included.

of $E_{\mathrm{th}}$ as a function of thickness for the two DLC films, along with the data reported previously for the PAC films, ${ }^{5}$ is presented in Fig. 1. It is apparent that over the range of thicknesses examined no discernible trend of $E_{\text {th }}$ with thickness is observed for the films deposited at the higher biases. The average value of $E_{\mathrm{th}}$ for the films deposited at $-90 \mathrm{~V}$ bias is 20 and $12 \mathrm{~V} / \mu \mathrm{m}$ for the films deposited at $-265 \mathrm{~V}$ bias. The explanation of observed thickness dependence of $E_{\text {th }}$ for the PAC films was based on the electrons at the back film $/ \mathrm{Si}$ heterojunction experiencing the applied field from the front surface and being accelerated towards it by the large internal field at the back contact. ${ }^{4,5}$ Such an explanation is plausible when it is noted that the defect density in these films is low, being $\sim 3 \times 10^{17} \mathrm{~cm}^{-3} .{ }^{9}$ These defects have been associated with the more conductive $s p^{2}$ clusters within the film. Field lines from the positively charged anode will terminate on these defects and result in localized screening of the field at the defect. However, the density of defects in the PAC films is too low for any global screening of the field to occur. In the case of the DLC films the defect density is 4 $\times 10^{18} \mathrm{~cm}^{-3}(-90 \mathrm{~V})$ and $1.8 \times 10^{20} \mathrm{~cm}^{-3}(-265 \mathrm{~V}) .{ }^{9}$ In the films with the higher defect densities, particularly in the film deposited at $-265 \mathrm{~V}$, a sufficiently large surface charge density is present, arising from the areal defect density of $\sim 10^{15} \mathrm{~cm}^{-2}$, to effectively screen the applied field to within a short surface layer. The film deposited at $-90 \mathrm{~V}$ bias is intermediate between the PAC films and film deposited at $-265 \mathrm{~V}$ bias.

Since the defects are believed to be located near to the Fermi level of the film ${ }^{9}$ the emission mechanism can be viewed as being Fowler-Nordheim (FN) tunneling into the vacuum. In this way the FE is determined by the properties of the film near to the front surface. The AFM images of these samples, not presented here, show that they possess a rms roughnesses of less than $0.6 \mathrm{~nm}$. If a FN emission process is present then it is necessary to explain the large enhancement factors, typically several hundred, required to lower a barrier by $4 \mathrm{eV} \cdot{ }^{5,7,10}$ In order to explain the origin of these large values of $\beta$, high resolution STM images of the

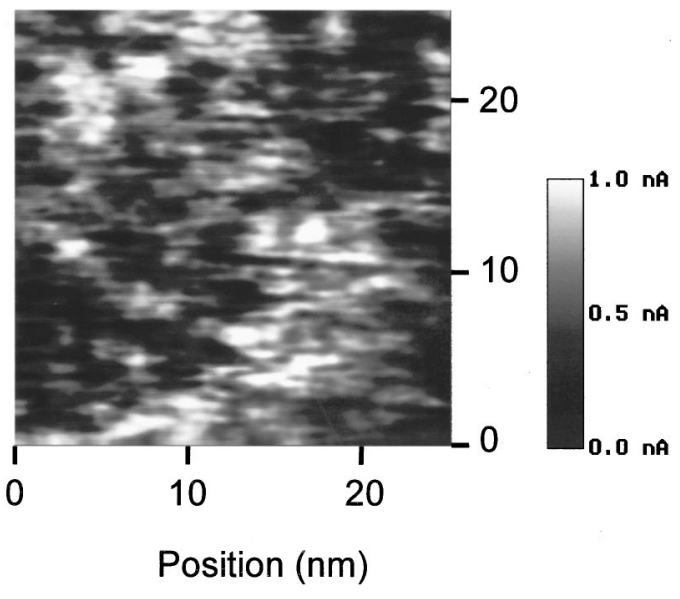

FIG. 2. High resolution STM image of the surface of the DLC film deposited at $-265 \mathrm{~V}$ bias showing regions of different conductivity.

DLC film grown at $-265 \mathrm{~V}$ have been performed and presented in Fig. 2. It is evident from the STM images that the conductivity of the film is not uniform. There are a number of localized high conductivity regions of $1-2 \mathrm{~nm}$ in dimension surrounded by a more insulating matrix. The STM results can be interpreted as resulting from a series of closely spaced localized conductive $s p^{2}$ regions which under the action of the applied field electrons may hop from one spatially localized cluster to another. ${ }^{9}$ In the continuous limit this will produce a conducting channel or filament which can extend through the bulk of the film. Field enhancement due to a free standing "protrusion" of length $h$ and radius $r$ can be approximated as $h / r .{ }^{11}$ If $r$ is taken to be in the range of $1-2$ $\mathrm{nm}$, obtained from the STM measurements, then for a $\beta$ value in the range of 100-300, say, $h$ must be at least $100-$ $600 \mathrm{~nm}$. Such a large well defined feature having a continuous pathway has never been observed using high resolution electron microscopy (HREM) (in either plan or cross section view). To date only well ordered microstructural region of about $1 \mathrm{~nm}$ has been observed using HREM. ${ }^{12}$ This leads us to conclude that an alternative source of enhancement must be present.

We propose the enhancement is due to the different dielectric and conductive properties of the $s p^{2}$ clusters when compared with surrounding $s p^{3}$ insulating matrix. Field enhancement near a single conductive dielectric sphere in a insulating matrix, is well known ${ }^{13}$ and originates from the dielectric mismatch between the two materials and typically can give small enhancements of 1-2. Chaumet and Dufour ${ }^{14}$ have considered the effects of the presence of two closely spaced dielectric spheres at an arbitrary orientation relative to the orientation of the applied field. Their calculations show that if two such conductive spheres, with the conductivity of gold, are placed $5 \mathrm{~nm}$ apart enhancements of 56 can be obtained which increases to over 400 for a $1 \mathrm{~nm}$ separation. Although the conductivity of the $s p^{2}$ clusters will not be as high as $\mathrm{Au}$, this classical electrostatic calculation shows that higher enhancement factors can be obtained by considering the effects of more than one conducting spheres near the surface. Relating this to nanoscale sized $s p^{2}$ clusters, the emission process for DLC films can be explained as follows: the high density of defects will act as to localize and attract the field lines from the anode. In a thin region near the sur-

license or copyright, see http://ojps.aip.org/aplo/aplcr.jsp 


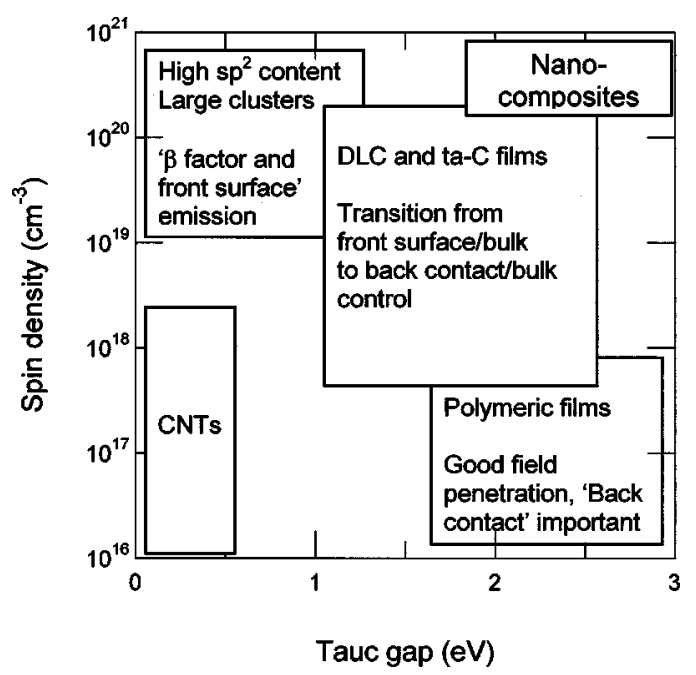

FIG. 3. Schematic representation indicating the main factors which govern electron emission from a range of amorphous carbon based materials. The magnitude of the spin density will indicate the extent of field penetration and the Tauc gap will indicate the size of the $s p^{2}$ clusters.

face of the film the inherent inhomogeneous nature present will result in different regions $\left(s p^{3}\right.$ or $\left.s p^{2}\right)$ experiencing different local fields. Additional field enhancement will occur if two (or more) $s p^{2}$ conductive clusters are nearby. Once the electrons are emitted from the clusters they may be replaced, via a hopping transport mechanism, by electrons from clusters deeper within the film. Such a description also helps explain the origin of the localized emission across the surface of the film which is dependent upon the local arrangement of the clusters near the surface.

By using these results it is possible to present a unified description of the emission process from $a-\mathrm{C}: \mathrm{H}$ based materials, as shown in Fig. 3, where the defect density versus optical Tauc gap is presented. The spin density may be used as an indicator of the extent of field penetration and the connectivity of the $s p^{2}$ clusters. ${ }^{9}$ The use of the connectivity within closely spaced clusters on a three-dimensional scale has recently been explained by Khan et al. which resulted in delocalized carriers transport in ion implanted $a-\mathrm{C}: \mathrm{H}$ films. ${ }^{15}$ The Tauc gap is a measure of the type of material and size of the clusters. ${ }^{3}$ The low defect density PAC films possess a wide optical gap in which a high degree of field penetration occurs and the emission process is one that is largely controlled by the hetrojunction at the back $a-\mathrm{C}: \mathrm{H} / \mathrm{Si}$ interface. The emission from high defect density DLC films is controlled by the front surface and internal enhancement due to closely spaced clusters and the ability of electrons to hop from one cluster to the next. This also applied to other high defect density materials such as undoped tetrahedral amorphous carbon ( $\mathrm{ta}-\mathrm{C}$ ) films. It is interesting to note that in the studies by Zhao et al. ${ }^{16}$ and Ilie et al. ${ }^{17}$ that no dependence of the threshold field with film thickness for $t a-\mathrm{C}$ films was observed, consistent with the model proposed here.

The FE properties of CNTs and carbon nanocomposite (CNC) films may also be explained within the confines of Fig. 3. CNTs have been located towards the low optical gap, low spin density region since these materials can be consid- ered to be rolled up graphite sheets with a small band gap. The defect density in these materials is a result of the presence of odd five- and sevenfold membered rings usually found towards the end of the CNTs. The emission from these materials is largely controlled by the high geometric enhancement. The FE properties of (CNC) films, consisting of nanotubes and nanoparticles embedded in $a$ - $\mathrm{C}$ matrix, have also been recently studied. ${ }^{18}$ These films would have a high band gap but, but unlike the PAC film, also have a high spin density due to the highly stressed nature of the deposited films. In this type of film it has been shown that the emission has been controlled by the heterojunction between the graphitic nanoparticles and the $a-\mathrm{C}: \mathrm{N}$ matrix as well as the high internal $\beta$ factor. In this way the FE from these materials is a combination of both the hetrojunction interface between the nanoparticles and the matrix as well as being controlled geometric enhancement effects.

In conclusion, the FE from different types of $a-\mathrm{C}: \mathrm{H}$ films have been examined. STM measurements of DLC films show variation of the conductivity across the film surface with nm sized conductive regions surrounded by a more insulating matrix. A model of field enhancement based upon the internal dielectric inhomogenities has been developed to explain the origin of the FE from DLC films. Extensions of this model to other forms of carbon based emitters have been proposed which may be used to explain many of the experimental observations from the different types of emitters.

This work has been sponsored by the EPSRC Grant No. GR/L77638. The authors would like to thank R. Khan for his assistance in the deposition of the films used in this study.

${ }^{1}$ See, for example, Proceedings of the First International Specialist Meeting on Amorphous Carbon, edited by S. R. P. Silva, J. Robertson, W. I. Milne, and G. A. J. Amaratunga (World Scientific, Singapore, 1998).

${ }^{2}$ J. E. Jaskie, MRS Bull. 21, 59 (1996).

${ }^{3}$ J. Robertson, J. Vac. Sci. Technol. B 17, 659 (1999).

${ }^{4}$ G. A. J. Amaratunga and S. R. P. Silva, Appl. Phys. Lett. 68, 2529 (1996).

${ }^{5}$ R. D. Forrest, A. P. Burden, S. R. P. Silva, L. K. Cheah, and X. Shi, Appl. Phys. Lett. 73, 3784 (1998).

${ }^{6}$ L. Zhang, T. Sakai, N. Sakuma, T. Ono, and K. Nakayama, Appl. Phys. Lett. 75, 3527 (1999).

${ }^{7}$ J. Robertson, Mater. Res. Soc. Symp. Proc. 498, 197 (1998).

${ }^{8}$ A. Ilie, A. C. Ferrari, T. Yagi, and J. Robertson, Appl. Phys. Lett. 76, 2627 (2000)

${ }^{9}$ J. D. Carey, R. D. Forrest, R. U. A. Khan, and S. R. P. Silva, Appl. Phys. Lett. 77, 2006 (2000)

${ }^{10}$ O. Gröning, O. M. Küttel, P. Gröning, and L. Schlapbach, Appl. Phys. Lett. 71, 2253 (1997).

${ }^{11}$ I. Brodie and P. R. Schwoebel, Proc. IEEE 7, 1006 (1994).

${ }^{12}$ C. A. Davis, S. R. P. Silva, R. E. Duninborkowski, G. A. J. Amaratunga, K. M. Knowles, and W. M. Stobbs, Phys. Rev. Lett. 75, 4258 (1995).

${ }^{13}$ See, for example, D. J. Griffiths, Introduction to Electrodynamics (Prentice-Hall, Englewood Cliffs, NJ, 1999), p. 146.

${ }^{14}$ P. C. Chaumet and J. P. Dufour, J. Electrost. 43, 145 (1998).

${ }^{15}$ R. U. A. Khan, J. D. Carey, S. R. P. Silva, B. J. Jones, and R. C. Barklie, Phys. Rev. B 63, 121201 (R) (2001).

${ }^{16}$ J. P. Zhao, Z. Y. Chen, X. Wang, T. S. Shi, and T. Yano, Appl. Phys. Lett. 76, 191 (2000).

${ }^{17}$ A. Ilie, A. Hart, A. J. Flewitt, J. Robertson, and W. I. Milne, J. Appl. Phys. 88, 6002 (2000).

${ }^{18}$ G. A. J. Amaratunga, M. Baxendale, N. Rupasinghe, I. Alexandrou, M. Chhowalla, T. Butler, A. Munindradasa, C. J. Kiley, L. Zhang, and T. Sakai, New Diamond Frontier Carbon Technol. 9, 31 (1999). 